\title{
Action observation training to improve motor function recovery: a systematic review
}

\author{
Elisabetta Sarasso ${ }^{1,2^{*}}$, Mariano Gemma', Federica Agosta², Massimo Filippi ${ }^{2}$ and Roberto Gatti ${ }^{3}$
}

\begin{abstract}
Following the discovery of Mirror Neuron System (MNS), Action Observation Training (AOT) has become an emerging rehabilitation tool to improve motor functions both in neurologic and orthopedic pathologies.

The aim of this study is to present the state of the art on the use of AOT in experimental studies to improve motor function recovery in any disease.

The research was performed in PubMed, PEDro, Embase, CINAHL and Cochrane Central Register of Controlled Trials (last search July 2015). Randomized controlled trials (RCTs) that analyse efficacy of AOT for recovery of motor functions, regardless of the kind of disease, were retrieved. The validity of the included studies was assessed using the Cochrane Collaboration tool for evaluating risk of bias.

Twenty RCTs were eligible. Four studies showed AOT efficacy in improving upper limb functional recovery in participants with chronic stroke, two studies in sub-acute ones and one in acute ones. Six articles suggested its effectiveness on walking performance in chronic stroke individuals, and three of them also suggested an efficacy in improving balance. The use of AOT was also recommended in individuals with Parkinson's disease to improve autonomy in activities of daily living, to improve spontaneous movement rate of self-paced finger movements and to reduce freezing of gait. Other two studies also indicated that AOT improves upper limb motor function in children with cerebral palsy. The last two studies, showed the efficacy of AOT in improving motor recovery in postsurgical orthopedic participants. Overall methodological quality of the considered studies was medium.

The majority of analyzed studies suggest the efficacy of AOT, in addition to conventional physiotherapy, to improve motor function recovery in individuals with neurological and orthopedic diseases. However, the application of AOT is very heterogeneous in terms of diseases and outcome measures assessed, which makes it difficult to reach, to date, any conclusion that might influence clinical practice.
\end{abstract}

Keywords: Action observation, Motor function recovery, Systematic review

\section{Background}

Mirror Neurons (MN) were described for the first time in the nineties by a group of researchers at the University of Parma, and localized in the ventral premotor cortex (F5 area) of macaques. [1] In this region, two types of neurons were identified: the canonical neurons, which respond during goal directed hand movement, and the visuo-motor mirror neurons, which are activated both when the monkey performs a particular motor gesture directed toward an object and when this action is seen

\footnotetext{
* Correspondence: sarasso.elisabetta@hsr.it

${ }^{1}$ Rehabilitation Department, San Raffaele Scientific Institute, Milan, Italy

${ }^{2}$ Neuroimaging Research Unit, INSPE, Division of Neuroscience, San Raffaele

Scientific Institute, Vita-Salute San Raffaele University, Milan, Italy

Full list of author information is available at the end of the article
}

without executing. The existence of MN in humans has been confirmed by studies performed with Transcranial Magnetic Stimulation (TMS) [2] and non-invasive neuroimaging techniques [3] that demonstrated the presence of classes of neurons that are compatible with those observed in macaques. In humans, $\mathrm{MN}$ have also been described in the rostral part of the inferior parietal lobule (IPL), whose properties appear to be similar to those of neurons in the premotor cortex. These two areas are connected together and form a network which is a part of the fronto-parietal circuit that organizes actions $[4,5]$. MN of humans also play an important role in understanding the intentions of other actions. Functional MRI (fMRI) studies indeed confirmed the same activation of MN both 
when the intent of the subject is easily understandable and when it is ambiguous [6].

The discovery that $\mathrm{MN}$ are involved in motor learning [7] has allowed the development of a new rehabilitation approach, called Action Observation Training (AOT), during which the patient is asked to carefully observe actions presented through a video-clip or performed by an operator, in order to try and imitate them after the observation. The purpose of AOT in the rehabilitation of individuals with lesions of the central nervous system (CNS) is to provide a tool to recover damaged cerebral networks [8] and take advantage to rebuild motor function despite impairments, as an alternative or complement to physiotherapy [9]. Several studies $[10,11]$ confirmed the hypothesis that the imitation of observed gestures lead to a reorganization of the primary motor cortex, contributing to the formation of motor memory of the observed action, physiological process underlying motor learning. The clinical relevance is easy to understand: when a patient is unable to perform movements because of neural damage or pain or imposed immobility, AOT offers the possibility to activate specific areas of the cerebral cortex, reinforcing intact cortical networks and facilitating the activation of the damaged ones, preventing changes in cortical reorganization that occur after inactivity and disuse [12]. Based on these findings, in the last ten years several studies on the clinical use of AOT have been published.

\section{Review}

\section{Objectives}

The aim of this study is to present a systematic review on the use of AOT in experimental studies to improve motor function recovery in any disease. It was decided to investigate the modality of application and the posology of this technique, the diseases on which it was applied, the objectives and the outcome measures used to assess its efficacy.

\section{Materials and methods}

\section{Inclusion and exclusion criteria}

Randomized controlled trials (RCTs) that focused on the effects of a period of AOT on motor rehabilitation were included. There was no restriction on disease, impairment and disability of the participants. The following selection criteria (PICO) were used: a randomized controlled trial design, a patient population including any kind of disease, a rehabilitative intervention focused on AOT, outcomes of motor function recovery. All the articles had to be available in English and full-text.

\section{Search strategy}

Pubmed (from 1950), PEDro (from 1929), Embase (from 1980), CINAHL (from 1982) and the Cochrane Central Register of Controlled Trials (from 1929) databases were electronically searched until July 2015. Three key terms action observation, rehabilitation, and motor function were used to generate a list of search terms, which were combined into a search strategy adapted to each database: (action OR motor OR movement) AND observation AND (training OR treatment OR therapy OR physical training OR movement execution OR rehabilitation OR neurorehabilitation) AND ((motor AND (function OR recovery OR learning OR activity OR ability)) OR (functional recovery). The extended version is available in Additional file 1.

\section{Study selection}

Among the articles found by the search (see flowchart in Fig. 1), 29 were selected according to the titles and abstracts by two independent reviewers (ES, MG). Reference lists of identified studies and published reviews were manually checked for additional RCTs. References retrieved by the electronical search were compared for duplicate entries and were manually cross-checked. Eligible papers were gathered in full-text, independently screened by the same reviewers. A third reviewer (RG) facilitated decision-making when there was disagreement.

\section{Assessment of risk of bias in included studies}

The risk of bias of the included studies was independently assessed by two review authors (ES and MG) using the Cochrane Collaboration's "Risk of bias" tool [13]. The assessment was achieved by assigning a judgment of 'low risk' of bias when bias was considered unlikely to have altered the results, 'high risk' of bias when the potential for bias weakened confidence in the results, or 'unclear risk' when there was some doubt about the effect of bias on the results. The following topics were assessed: description of randomization, allocation concealment, blinding, completeness of outcome data, and selective reporting [13]. Considering the nature of the intervention, blinding of the physiotherapists administering the AOT was impractical,

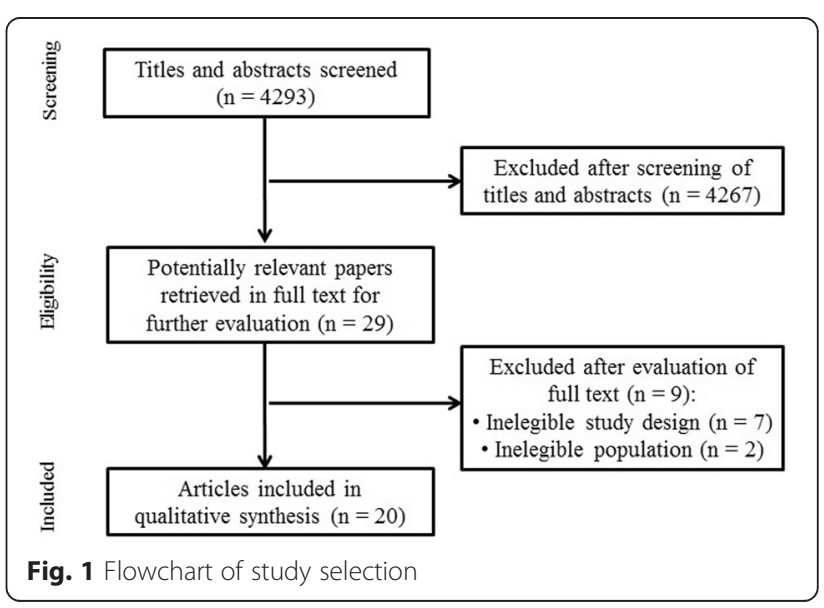


so only outcome assessor and participant blinding was considered.

\section{Results}

Two of the 29 articles were excluded because they were study protocols [14, 15], five because they were not RCTs [16-20] and two because they were about AOT in healthy subjects (young or elderly) [21, 22]. The letter Buccino et al. 2011 was included despite not being a full
RCT research paper, since as authors of the letter we can confirm it fulfilled the necessary requirements to be included in our analysis. As a result of the screening, 20 articles were included in the review (see Table 1).

\section{Characteristics of included studies}

The characteristics of included studies are summarized in the Table 2 and extensively reported in the Additional file 2 .

Table 1 Included articles ordered by year of publication

\begin{tabular}{|c|c|c|c|}
\hline Title & Authors & Journal & Year \\
\hline $\begin{array}{l}\text { A mirror therapy-based action observation protocol to improve motor } \\
\text { learning after stroke. }\end{array}$ & $\begin{array}{l}\text { Harmsen WJ, Bussmann JB, Selles RW, } \\
\text { Hurkmans HL, Ribbers GM. }\end{array}$ & $\begin{array}{l}\text { Neurorehabil Neural } \\
\text { Repair }\end{array}$ & 2015 \\
\hline $\begin{array}{l}\text { The effects of action observation gait training on the } \\
\text { static balance and walking ability of stroke patients. }\end{array}$ & Park EC, Hwangbo G. & J Phys Ther Sci & 2015 \\
\hline $\begin{array}{l}\text { Effects of purposeful action observation on kinematic patterns } \\
\text { of upper extremity in individuals with hemiplegia. }\end{array}$ & Kim E, Kim K. & J Phys Ther Sci & 2015 \\
\hline $\begin{array}{l}\text { The effect of action observation training on knee joint function } \\
\text { and gait ability in total knee replacement patients. }\end{array}$ & Park SD, Song HS, Kim JY. & J Exerc Rehabil & 2014 \\
\hline $\begin{array}{l}\text { Action observation therapy in the sub-acute phase promotes } \\
\text { dexterity recovery in right-hemisphere stroke patients. }\end{array}$ & Sale P, Ceravolo MG, Franceschini M. & Biomed Res Int & 2014 \\
\hline $\begin{array}{l}\text { Clinical feasibility of action observation training for walking function } \\
\text { of patients with post-stroke hemiparesis: a randomized controlled trial. }\end{array}$ & Park HR, Kim JM, Lee MK, Oh DW. & Clin Rehabil & 2014 \\
\hline $\begin{array}{l}\text { Action observation training for functional activities } \\
\text { after stroke: A pilot randomized controlled trial. }\end{array}$ & Kim JH, Lee BH. & NeuroRehabilitation & 2013 \\
\hline $\begin{array}{l}\text { The effects of action observational training on walking ability in } \\
\text { chronic stroke patients: a double-blind randomized controlled trial. }\end{array}$ & Bang DH, Shin WS, Kim SY, Choi JD. & Clin Rehabil & 2013 \\
\hline The effects of additional action observational training for functional & Park CS, Kang KY. & J Phys Ther Sci & 2013 \\
\hline
\end{tabular}
electrical stimulation treatment on weight bearing, stability and gait velocity of hemiplegic patients.

Reduction of bradykinesia of finger movements by a single session of action observation in Parkinson disease.

Drinking behavior training for stroke patients using action observation and practice of upper limb function.

Observation-to-imitate plus practice could add little to physical therapy benefits within 31 days of stroke: translational randomized controlled trial.

Randomized trial of observation and execution of upper extremity actions versus action alone in children with unilateral cerebral palsy.

Improving upper limb motor functions through action observation treatment: a pilot study in children with cerebral palsy.

Clinical relevance of action observation in upper-limb stroke rehabilitation: a possible role in recovery of functional dexterity. A randomized clinical trial.

Clinical feasibility of action observation based on mirror neuron system in walking performance on post stroke patients.

Action observation treatment improves autonomy in daily activities in Parkinson's disease patients: results from a pilot study.

Action observation treatment improves recovery of postsurgical orthopedic patients: evidence for a top-down effect?

Action observation improves freezing of gait in patients with Parkinson's disease.

Action observation has a positive impact on rehabilitation of motor deficits after stroke.
Pelosin $E$, Bove $M$, Ruggeri $P$, Avanzino L, Abbruzzese G.

Lee D, Roh H, Park J, Lee S, Han S.

Cowles T, Clark A, Mares K, Peryer G, Stuck R, Pomeroy V.

Sgandurra G, Ferrari A, Cossu G, Guzzetta A, Fogassi L, Cioni G.

Buccino G, Arisi D, Gough P, Aprile D, Ferri C, Serotti L, Tiberti A, Fazzi E.

Franceschini M, Ceravolo MG, Agosti M, Cavallini Neurorehabil Neural 2012 P, Bonassi S, Dall'Armi V, Massucci M, Schifini F, Repair Sale P.

Kim JS, Kim K.

Buccino G, Gatti R, Giusti MC, Negrotti A, Rossi A, Calzetti S, Cappa SF.

Bellelli G, Buccino G, Bernardini B, Padovani A, Trabucchi M.

Pelosin E, Avanzino L, Bove M, Stramesi P, Nieuwboer A, Abbruzzese $G$.

Ertelt D, Small S, Solodkin A, Dettmers C, McNamara A, Binkofski F, Buccino G.
J Phys Ther Sci

Neurorehabil Neural 2013 Repair

J Phys Ther Sci 2013

Neurorehabil Neural 2013 Repair

Neurorehabil Neural 2013 Repair

Dev Med Child 2012

Mov Disord

Arch Phys Med

Rehabil

Neurorehabil Neural 2010 Repair

Neuroimage 
Table 2 Summary of included studies

\begin{tabular}{|c|c|c|c|}
\hline Study & Participants & Intervention & Outcome measures \\
\hline Harmsen W. J. et al. [24] & $\begin{array}{l}37 \text { participants in the chronic } \\
\text { stage after stroke } \\
\text { Exp: } n=18(9 \mathrm{~F}) \\
\text { Age: } 57(\mathrm{SD} 10.4) \\
\text { Con: } \mathrm{n}=19(6 \mathrm{~F}) \\
\text { Age: } 60 \text { (SD 8.8) }\end{array}$ & $\begin{array}{l}\text { Exp: observation of the upper-arm reaching } \\
\text { movements similar to what patients would } \\
\text { see in the mirror during mirror therapy } \\
(3+1+1+1 \text { min })+\text { execution of reaching } \\
\text { movements }(30+20+20 \text { reps) } \\
\text { Con: observation of a slideshow } \\
\text { with static photographs of landscapes } \\
\text { ( } 3+1+1+1 \text { min) + execution of reaching } \\
\text { movements ( } 30+20+20 \text { reps) }\end{array}$ & $\begin{array}{l}\text { Upper limb Kinematics: movement time and } \\
\text { acceleration during a reaching movement. }\end{array}$ \\
\hline Park E.C. et al. [33] & $\begin{array}{l}40 \text { chronic stroke individuals } \\
\text { Exp: } n=20(10 \mathrm{~F}) \\
\text { Age }=51.15(\mathrm{SD} 14.81) \\
\text { Con: } n=20(9 \mathrm{~F}) \\
\text { Age }=48.65(\text { SD 12.81) }\end{array}$ & $\begin{array}{l}\text { Both: } 30 \text { min PT, } 5 / w k \times 8 w k \\
\text { Exp: observation of } 3 \text {-minute videos of walking } \\
(3 \text { videos) }+ \text { walking training for } 20 \text { minutes } \\
\text { ( } 30 \text { min, } 5 / w k \times 8 w k) \\
\text { Con: observation of } 3 \text {-minute landscapes videos } \\
\text { ( } 3 \text { videos) + walking training for } 20 \text { minutes } \\
(30 \text { min, 5/wk } \times 8 \text { wk) }\end{array}$ & $\begin{array}{l}\text { Balance ability = analysis system using biofeedback } \\
\text { (LOS, SS, SA) } \\
\text { Gait ability = TUG, 10MWT }\end{array}$ \\
\hline Kim E. et al. [25] & $\begin{array}{l}12 \text { stroke individuals } \\
\text { Exp: } n=6 \\
\text { Con: } n=6\end{array}$ & $\begin{array}{l}\text { Exp: traditional occupational treatment } \\
\text { + purposeful action observation training } \\
\text { program ( } 30 \text { min, } 5 / w k \times 6 w k) \\
\text { Con: traditional occupational treatment } \\
\text { + topological treatment in which they } \\
\text { performed purposeful action observation } \\
\text { program assignments without actually } \\
\text { observing the purposeful actions } \\
\text { (30 min, 5/wk } \times 6 \mathrm{wk})\end{array}$ & $\begin{array}{l}\text { Upper limb Kinematics: average velocity, trajectory } \\
\text { ratio, motion angle }\end{array}$ \\
\hline Sale et al. [28] & $\begin{array}{l}67 \text { sub-acute stroke } \\
\text { individuals ( } 26 \text { F) } \\
\text { Age }=66.5 \text { (SD 12.7) }\end{array}$ & $\begin{array}{l}\text { Exp: observation of } 3 \text {-minute videos } \\
\text { of manual tasks ( } 3 \text { videos) }+ \text { execution } \\
\text { of observed movement for } 2 \text { minutes } \\
(3 \text { times })(2 \times 15 \text { min, } 5 / w k \times 4 w k) \\
\text { Con: observation of } 5 \text { static images } \\
\text { displaying objects ( } 3 \text { minutes) + execution } \\
\text { of same movement as exp group for } \\
2 \text { minutes ( } 3 \text { times) }(2 \times 15 \text { min, } \\
5 / w k \times 4 w k)\end{array}$ & $\begin{array}{l}\text { Manual dexterity: BBT } \\
\text { Motor impairment: FMA } \\
\text { Follow up }=4-5 \mathrm{mth}\end{array}$ \\
\hline Park H.R. et al. [34] & $\begin{array}{l}21 \text { chronic stroke individuals } \\
\text { Exp: } \mathrm{n}=11 \text { (3 F) } \\
\text { Age }=55.9(\mathrm{SD} 9.1) \\
\text { Con: } \mathrm{n}=10(3 \mathrm{~F}) \\
\text { Age }=54.8(\mathrm{SD} 12.22)\end{array}$ & $\begin{array}{l}\text { Exp: observation of } 10 \text { minutes of video } \\
\text { demonstrating four tasks for functional } \\
\text { walking }+20 \text { min walking training } \\
\text { ( } 30 \text { min, 3/wk } \times 4 \text { wk) } \\
\text { Con: } 10 \text { minutes of different landscape } \\
\text { images }+20 \text { min walking training } \\
\text { ( } 30 \text { min, } 3 / w k \times 4 w k \text { ) }\end{array}$ & $\begin{array}{l}\text { Gait ability = } 10 \text { MWT, F8WT, DGI, Gait symmetry } \\
\text { scores }\end{array}$ \\
\hline Kim J.H. et al. [31] & $\begin{array}{l}30 \text { chronic stroke individuals } \\
\text { Exp AOT: } n=9(2 \mathrm{~F}) \\
\text { Age }=55.3(\text { SD } 12.1) \\
\text { Exp Mi: } n=9(3 \mathrm{~F}) \\
\text { Age }=54.8 \text { (SD 8.8) } \\
\text { Con: } n=9(2 \mathrm{~F}) \\
\text { Age }=59.8 \text { (SD 8.9) }\end{array}$ & $\begin{array}{l}\text { Exp AOT: observation of } 20 \text { minutes-video } \\
\text { followed by physical training ( } 10 \text { minutes) } \\
\text { ( } 30 \text { min, } 5 / w k \times 4 w k \text { Exp Mi: } 20 \text { minutes } \\
\text { of Mi followed by physical training for } \\
10 \text { minutes( } 30 \text { min, } 5 / w k \times 4 w k) \\
\text { Con: Physical training ( } 30 \text { min, } \\
\text { 5/wk } \times 4 \text { wk) }\end{array}$ & $\begin{array}{l}\text { Gait ability = TUG, WAQ, FAC } \\
\text { Gait kinematics = Spatiotemporal gait parameters } \\
\text { Balance = FRT }\end{array}$ \\
\hline
\end{tabular}

Exp: traditional occupational treatment

+ purposeful action observation training

program (30 min, 5/wk x 6wk)

ing the purposeful actions

Exp: observation of 3-minute videos

f manual tasks (3 videos) + execution

forved movement for 2 minutes

(3imes) $(2 \times 15 \mathrm{~min}, 5 / \mathrm{wk} \times 4 \mathrm{wk})$

displaying objects ( 3 minutes) + execution

of same movement as exp group for

$5 / w k \times 4 w k$

Exp: observation of 10 minutes of video rating four tasks for functiona

m walking training

Exp AOT: observation of 20 minutes-video

wed by physical training (10 minutes)

$5 / w k \times 4 w k)$ 
Table 2 Summary of included studies (Continued)

30 chronic stroke individuals

Exp: $\mathrm{n}=15$ (6 F)

Age $=64.1($ SD 6.35)

Con: $n=15(7 \mathrm{~F})$

Age $=58.9($ SD 6.03)

Park C.S. et al. [35] 20 chronic stroke individuals Exp: $n=10(4 \mathrm{~F})$

Con: $n=10(5 F)$

Lee D. et al. [26]

33 chronic stroke individuals

Action observation group: $\mathrm{n}=8$ (3 F),

Age: 63 (SD 3.7)

Action practice group:

$n=9$ (4 F) Age: 62 (SD 1.5)

Combination group:

$\mathrm{n}=9$ (4 F) Age: 61 (SD 2.3)

Control group:

$n=7$ (4 F) Age: 60 (SD 5.9)

Cowles et al. [29]

29 acute stroke individuals

Exp: $\mathrm{n}=15$ (7 F)

Age $=78.8($ SD 8.1)

Con: $n=14(5 F)$

Age $=75.6($ SD 12.4)

Franceschini

et al. [27]

102 sub-acute stroke individuals

Exp: $\mathrm{n}=53$ (20 F)

Age $=67.0($ SD 12.4)

Con: $\mathrm{n}=49$ (21 F)

Age $=65.7(S D$ 11.9)

Kim JS et al. [32]

30 chronic stroke individuals

Exp: $\mathrm{n}=15$,

Age $=64.1($ SD 8.3)

Age $=65.5(7.7)$

Ertelt et al. [23]

15 chronic stroke individuals Exp: $\mathrm{n}=7$ (2 F)

Age $=57.16($ SD 8.73)
Exp: treadmill training (30 $\mathrm{min})$ after watching

the treadmill video (9 $\mathrm{min}$ ) (40 min

$5 / w k \times 4 w k)$

Con: treadmill training (30 min) after

watching the nature video ( $9 \mathrm{~min})$.

(40 min, 5/wk $\times 4$ wk)

Both: functional electrical stimulation

treatment.

Exp $=$ observation of a video on gait

for 15 minutes. The video showed

walking on flat ground, slopes and stairs. (15 min, 5/wk x 4wk)

The action observation group watched

a video of the task (10 minutes), the

action practice group performed the

action (10 minutes), the combined

action observation-action practice

group watched the video of the task

$(5$ minutes) and practiced the action

(5 minutes), and the control group

(5 minutes), and the control grour

did not perform either action

observation or action practice.

Exp: $3 \times 8$ min imitation of therapist performing functional activities with upper limb (2 x 30 min, 15 consecutive working days)

Con: no therapy in addition to

conventional physiotherapy

Exp: observation of 3-minute videos

of manual tasks (3 videos) + execution

of observed movement for 2 minutes

(3 times) (2 $\times 15$ min, 5/wk $\times 4 w k)$

Con: observation of 5 static images

displaying objects (3 minutes)+

execution of same movement as

exp group for 2 minutes (3 times)

$(2 \times 15 \mathrm{~min}, 5 / \mathrm{wk} \times 4 \mathrm{wk})$

Both: 30 min PT

Exp: observation of 2-minute videos

of walking ( 5 videos) + walking training

for 10 minutes

Con: 10 minutes of video in

which they were taken through

a progressive relaxation program

(stretching)

(90 min $\times 18$ consecutive working days)

Exp: observation of 6-minute videos

of daily life hand and arm actions

3 videos) + execution of observed
Gait ability = TUG, 10MWT, 6MW

Gait kinematics $=$ Knee angle in

swing phase during walking

Balance ability: Weight Distribution, Stability Index Gait kinematics: Gait Velocity

Upper-limb functional dexterity: number of times the full drinking action was performed in one minute Follow up $=1$ wk

Functional use of upper limb = ARAT

Upper-limb functional dexterity: FAT, BBT, FIMM Motor impairment: FMA

Follow up $=4-5 \mathrm{mth}$

Gait kinematics: Spatiotemporal gait parameters (including walking speed)

Upper-limb functional dexterity: WMFT, FAT Subjective scale: SIS

Follow up $=8 \mathrm{w}$ 
Table 2 Summary of included studies (Continued)

\begin{tabular}{|c|c|c|c|}
\hline & $\begin{array}{l}\text { Con: } n=8(2 F) \\
\text { Age }=55.40(\text { SD 10.77) }\end{array}$ & $\begin{array}{l}\text { movement for } 6 \text { minutes ( } 3 \text { times) } \\
\text { Con: observation of sequences of } \\
\text { geometric symbols and letters + } \\
\text { execution of same movement as } \\
\text { exp group for } 6 \text { minutes ( } 3 \text { times) }\end{array}$ & \\
\hline Pelosin et al. [38] & $\begin{array}{l}38 \text { individuals with } P D(21 \mathrm{~F}) \\
\text { Age }=67.4(\mathrm{SD} 7.4) \\
14 \text { matched healthy controls }(7 \mathrm{~F})\end{array}$ & $\begin{array}{l}\text { VIDEO group: observation of a } \\
6 \text {-minute video clip showing } \\
\text { repetitive finger movements } \\
\text { paced at } 3 \mathrm{~Hz} \\
\text { ACOUSTIC group: listening to } \\
\text { an acoustic cue paced at } 3 \mathrm{~Hz} \\
\text { for } 6 \text { minutes. } 8 \text { participants with } \\
\text { PD were recruited for a sham } \\
\text { intervention, watching a } 6 \text {-minute } \\
\text { video representing a static hand. }\end{array}$ & $\begin{array}{l}\text { Spontaneous movement rate (SMR) of self- } \\
\text { paced finger movements. } \\
\text { Follow up }=2 \text { days }\end{array}$ \\
\hline Buccino et al. [36] & $\begin{array}{l}15 \text { PD individuals } \\
\text { Exp: } n=7 \\
\text { Age }=68(2 \mathrm{~F}) \\
\text { (min-max: } 59-80) \\
\text { Con: } n=8 \\
\text { Age }=73.5(3 \mathrm{~F}) \\
\text { (min-max: } 67.5-76.5)\end{array}$ & $\begin{array}{l}\text { Exp: observation and subsequently } \\
\text { execution of different daily actions } \\
\text { presented through video clips } \\
\text { Con: observation of video clips } \\
\text { with no motor content and } \\
\text { subsequently performance of } \\
\text { the same actions as exp group }\end{array}$ & Autonomy in ADL: UPDRS, FIM \\
\hline Pelosin et al. [37] & $\begin{array}{l}18 \text { individuals with PD (8 F) } \\
\text { Exp: } n=9 \\
\text { Age }=68.8 \text { (SD 4.1) } \\
\text { Con: } n=9 \\
\text { Age }=70.2 \text { (SD 6.8) }\end{array}$ & $\begin{array}{l}\text { Exp: observation of } 6 \text {-minute } \\
\text { videos of walking tasks } \\
\text { ( } 4 \text { videos) + execution of } \\
\text { the same motor training } \\
\text { ( } 36 \text { minutes) (60 min, } \\
3 / w k \times 4 w k \text { ) } \\
\text { Con: observation of landscape } \\
\text { videos + execution of same } \\
\text { exercises in the exact order } \\
\text { and for the same amount of } \\
\text { time than AOT group ( } 60 \text { min, } \\
\text { 3/wk } \times 4 \text { wk) }\end{array}$ & $\begin{array}{l}\text { FoG frequency and severity: FoG-Q, FoG-diary } \\
\text { Disease severity: H\&Y } \\
\text { Motor impairment: UPDRSIII } \\
\text { Gait ability = TUG, 10MWT } \\
\text { Balance ability: BBS, Tinetti score } \\
\text { Quality of life: PDQ-39 } \\
\text { Follow up }=4 \text { wk }\end{array}$ \\
\hline Sgandurra et al. [40] & $\begin{array}{l}24 \text { cerebral palsy children } \\
\text { Exp: } n=12(4 \mathrm{~F}) \\
\text { Age } 9.48(\text { SD 2.12) } \\
\text { Con: } n=12(4 \mathrm{~F}) \\
\text { Age } 9.94 \text { (SD 2.77) }\end{array}$ & $\begin{array}{l}\text { Exp: observation of } 3 \text { minutes- } \\
\text { videos representing upper limb } \\
\text { actions + execution of the same } \\
\text { motor tasks ( } 3 \text { minutes)( } 60 \text { min x } \\
15 \text { consecutive working days) } \\
\text { Con: observation of computer } \\
\text { games + execution of the same } \\
\text { motor tasks as exp group ( } 60 \text { min x } \\
15 \text { consecutive working days) }\end{array}$ & $\begin{array}{l}\text { Upper limb function = AHA, MUUL } \\
\text { Manual ability = ABILHAND-Kids questionnaire } \\
\text { Follow up }=1-8-24 \text { wk }\end{array}$ \\
\hline Buccino et al. [39] & $\begin{array}{l}24 \text { cerebral palsy children } \\
\text { Exp: } n=8(4 \mathrm{~F}) \\
\text { Con: } n=7(2 \mathrm{~F})\end{array}$ & $\begin{array}{l}\text { Exp: observation of } 9-12 \text { minutes- } \\
\text { videos representing upper limb } \\
\text { actions }+ \text { execution of the same } \\
\text { motor tasks (6-8 minutes) } \\
\text { (5/wk } \times 3 \text { wk) }\end{array}$ & Upper limb function = MaS \\
\hline
\end{tabular}

ough video clips

the same actions as exp group

exp: observation of 6-minute

videos of walking tasks

the same motor training

on: observation of landscape

videos + execution of same

xercises in the exact order

for the same amount of $3 / w k \times 4 w k)$

xp: observation of 3 minutes-

ideos representing upper limb

exp group (60 min

$5 / w k \times 3 w k)$ 
Table 2 Summary of included studies (Continued)

$\begin{array}{ll}\text { Park S.D. } & 18 \text { individuals with TKR } \\ \text { et al. [34] } & \text { Exp: } n=9 \\ & \text { Age }=72.67 \text { (SD 12.25) } \\ & \text { Con: } n=9 \\ & \text { Age }=70.56 \text { (SD 10.98) }\end{array}$

Con: observation of history, geography

or science videos (9-12 minutes) +

execution of the same motor tasks

as exp group (6-8 minutes)

(5/wk $\times 3 w k)$

Both: 30 minutes of gait exercise and

treadmill

Exp: observation of a 10 Iminutes-

video clip showing daily actions +

execution (30 minutes) of the same

actions (3/wk $\times 3 w k)$ Con: execution

of the same actions as patients in

the AOT group (30 minutes)

$$
(3 / w k \times 3 w k)
$$

Bellelli et al. [41]

60 individuals (hip fractures or hip or knee replacement) Both: 60 min conventional postExp: $\mathrm{n}=30$ (21 F)

Age $=71.9($ SD 8.4)

Con: $\mathrm{n}=30$ (16 F)

orthopedic rehabilitation

Exp: observation of 8 minutes-

video clips showing daily actions

(3 videos) + execution of the same

actions (6/wk $\times 3 w k)$

Con: observation geographic

documentary + execution of the

same actions as patients in the $\mathrm{AO}$

group (6/wk $\times 3 w k)$

Exp experimental group, Con control group, $F$ female, wk week, reps repetitions, PT physical therapy, LOS limit of stability, SS sway speed, SA sway area, TUG timed up and go, 10MWT 10-metre walk test, BBT box and block test, FMA Fugl-Meyer Assessment, F8WT figure-of-8 walk test, DGI dynamic gait index, AOT action observation training, Mi motor imagery, WAQ walking ability questionnaire, FRT functional reach test, FAC functional ambulation categories, $6 M W T$ 6-minute walking test, $M I$ motricity index, ARAT action research arm test, FIMM functional independence measure motor score, WMFT wolf motor funtion test, SIS stroke impact scale, $P D$ Parkinson's disease, ADL activity of daily living, FoG-Q freezing of gait questionnaire, H\&Y Hoen and Year scale, UPDRS unified Parkinson's disease rating scale, BBS Berg balance scale, $P D Q-39$ Parkinson's disease questionnaire 39, AHA assisting hand assessment, MUUL Melbourne assessment of unilateral upper limb function, MaS Melbourne assessment scale, TKR total knee replacement, WOMAC Western Ontario and McMaster Universities osteoarthritis index 


\section{Participants}

Seven studies involved stroke individuals with upper limb impairment: 97 chronic stroke subjects in four studies [23-26], 169 sub-acute stroke participants in two studies [27, 28], and 29 acute stroke individuals in one study [29]. Six articles [30-35] investigated a population of 171 chronic stroke subjects with walking deficits, and three [33-35] of them also balance deficits $(N=90)$. The use of AOT was also explored in three samples of 15 [36], 18 [37] and 38 [38] participants with Parkinson's disease. Other two studies analyzed a population of 48 children with cerebral palsy $[39,40]$ with upper limb motor impairment. Finally, two studies [41, 42] investigated the effect of AOT in 78 postsurgical orthopedic individuals.

\section{Intervention}

In the experimental (AOT) group, $30 \%$ of studies $[25,32,33,35,41,42]$ combined AOT to standard rehabilitation. The majority of studies resulted in an equal treatment time between the experimental and control groups. All the studies administered AOT through videos with the exception of one [29] in which subjects had to imitate actions performed by a physiotherapist. The characteristics of the intervention expressed as mean values (range) were: 12.4 min of AOT for each session (5-30); $7.4 \mathrm{~min}$ for each video; $16.9 \mathrm{~min}$ of observed actions performance (5-36); 6 sessions a week (3-10); total duration of treatment $=16.2$ days $(1-40)$. In all studies, with the except of three [29, 31, 32], the control group performed the same actions of the experimental group for the same amount of time. The only difference was that the control group watched videos without motor contents (landscapes, documentaries, geometric shapes, etc.), with the except of four studies [29, 31, 32, 42]: in one [32] of them the videos showed stretching exercises, in the other studies $[29,31,35,42]$ the control group did not see any video. Only one study [26] compared the effects of $10 \mathrm{~min}$ of "standard" AOT (5 min video, 5 min repetition) relative to $10 \mathrm{~min}$ of observation or $10 \mathrm{~min}$ of imitation alone. Finally, a study [38] investigated the different effects of a single-session of action observation without execution, relative to both a single-session of listening an acoustic cue and a single-session of static video, in improving spontaneous movement rate of self-paced finger movements in participants with Parkinson's disease.

\section{Outcome measures}

In keeping with the heterogeneous patient population included into the studies, also the outcomes used were very mixed. All outcomes are listed in Table 2 and in Additional file 2. Only RCTs including individuals with stroke showed a consistency in the use of outcome measures. Indeed, two studies $[27,28]$ used the Box and Block test and two [23, 27] the Frenchay Arm test to assess upper limb functional dexterity in sub-acute/chronic stroke participants, and three studies [30, 31, 33] used the Time Up and Go test (TUG) and three [30, 33, 34] the 10 $\mathrm{m}$ walking test (10MWT) to assess the walking ability in chronic subjects. Overall, in the 20 detected articles, 37 outcomes were administered.

\section{Quality}

The score on the risk of bias [13] achieved by each of the included studies are presented in Fig. 2. The overall quality of RCTs was medium. Eight of the RCTs reported a good randomization procedure $[29,32,34,37-40,42]$, while the others were 'unclear' $[23,25,26,30,31,33,35,36,41]$ or 'high risk' [24, 27, 28]. Only five studies reported a good allocation concealment [24, 29-31, 40], the other studies were 'unclear'. Only three studies reported the blinding of the participants [30, 39, 40]. In addition, 12 RCTs reported that the outcome assessors were blinded [26-30, 34, 36-42], while the others were 'unclear' [23-25, $31-33,35,42]$. 15 studies [24-26, 28-31, 34, 35, 37-42] reported the short term withdrawals and the reasons for these dropouts, but only five reported information about the long term withdrawals [26, 28, 37, 38, 40] (all the studies were analyzed on a per protocol basis). Five RCTs did not report a good selective outcome reporting $[23,25,27,32,36]$.

\section{Efficacy of AOT}

The studies included in this review suggest the efficacy of AOT in improving motor functions both in neurologic and orthopedic diseases. Thirteen articles [23-35] investigated the effectiveness of AOT in post-stroke rehabilitation. Among them, four studies [23-26] showed the efficacy of AOT in improving upper limb functional dexterity in individuals with chronic stroke and two studies in sub-acute subjects [27, 28]. In particular, one study [26] investigated the effects of "standard" AOT (combination of observation and imitation) relative to observation without imitation or execution without observation and to a control group in improving upper limb functional dexterity. This study [26] showed that all the experimental groups (combination, observation and imitation) clinically improved relative to the control group, while no clear difference emerged between the experimental groups. Only one study [29] including acute stroke participants showed a better recovery of functional dexterity in the group performing the conventional therapy. Six articles [30-35] suggest AOT efficacy on walking performance in subjects with chronic stroke and three $[31,33,35]$ of them also suggested an efficacy in improving balance. AOT is also recommended in individuals with Parkinson's disease to improve autonomy in activities of daily living (ADL) [36], to improve spontaneous movement rate of self-paced finger movements [38], and to reduce 


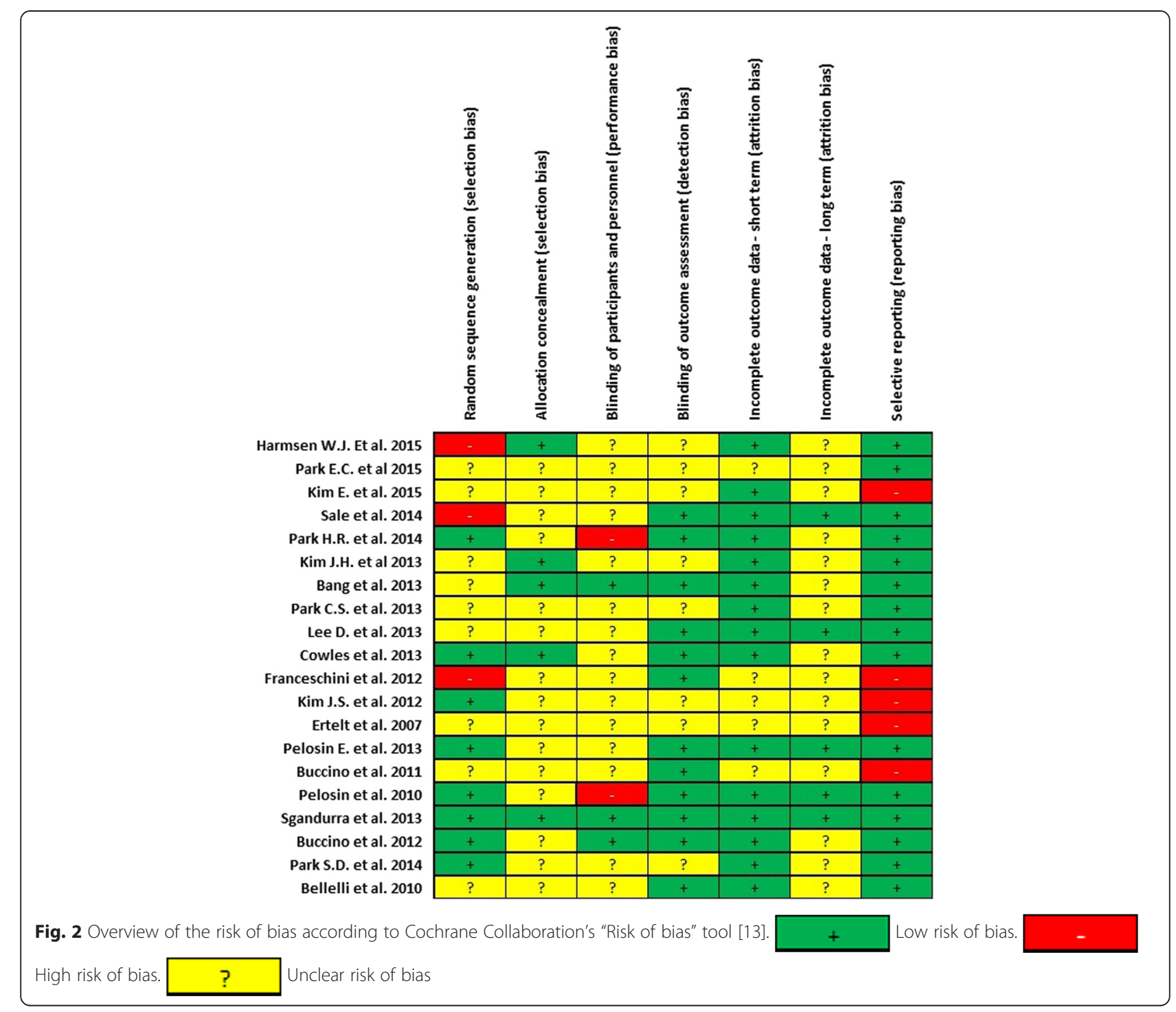

freezing of gait [37]. Two studies [39, 40] also indicate that AOT improves upper limb motor function in children with cerebral palsy. AOT seems to be effective in improving autonomy in ADL and balance in postsurgical orthopedic subjects [41] (hip fractures or hip or knee replacement) and to enhance knee joint function after total knee replacement [42].

\section{Discussion}

Twenty RCTs were included in this systematic review. The analyzed studies investigated the effects of AOT in improving different motor abilities in diseases like stroke, Parkinson's disease, cerebral palsy, and postsurgical orthopedic conditions, for a total of 663 subjects. The majority of the studies suggested the efficacy of AOT to improve motor function both in neurologic and orthopedic diseases.
The samples recruited in the most of the RCTs was relatively small and, overall, the quality of the studies was medium. The analysis about the quality highlighted the need to better specify the procedures for allocation concealment, handling of missing data, and blinding of study participants. Lack of uniformity on duration, and frequency of treatments also emerges from included studies, making it difficult to define an optimal posology; the most used duration of a single video is between 3 and $10 \mathrm{~min}$. In keeping with these results and our personal experience, videos lasting 5-6 min seem to be the most reasonable approach to obtain a good balance between individual sustained attention and training efficacy. One month is the most frequent duration of training. In individuals with Parkinson's disease, the frequency of 3 sessions a week has been suggested to be better than a continuous training because interval times 
might be necessary for learning consolidation in these subjects [43]. Further studies are needed to determine the optimal frequency, intensity and time of AOT.

Although the studies were too heterogeneous to be pooled, it is interesting to highlight that AOT has an effect in improving motor function regardless of the disease and the severity of motor impairment. Indeed, this approach can be easily adapted to many different conditions, is inexpensive, and can be easily tailored to specific needs of individuals.

No study reported data about the Minimal Clinical Important Difference (MCID) for any outcome measure. We obtained available MCID values from Rehabmeasure.org [44, 45]. Two studies aimed at improving walking ability in chronic stroke individuals achieved mean outcome values equal or greater than the corresponding MCID, i.e., one [33] in the 10MWT $(0.16 \mathrm{~m} / \mathrm{s})$ and the other one in the $6 \mathrm{~min}$ walking test $(89.6 \mathrm{~m})$ and 10MWT $(0.36 \mathrm{~m} / \mathrm{s})$ [30]. Another study [27], that was designed to investigate the effect of AOT on upper limb functional dexterity in sub-acute stroke participants, achieved a mean Functional Independence Measure score greater than the MCID at two follow-up visits (22.3 and 32.2, respectively).

Probably, the reason why AOT is helpful in addition to a conventional motor training is that it has been shown to facilitate motor learning and the building of a motor memory. It is well known that AOT recruits areas of motor network and MNS such as the ventral premotor cortex, inferior frontal gyrus and IPL, that are activated both during the observation of actions which are part of the motor repertoire of the observer [46] and also in acquiring new motor skills [11]. MNS plays an important part in motor learning [47] that is defined as "a set of processes associated with practice, leading to relatively permanent changes in the capability for movement". Thus, AOT can be considered as a cognitive tool to improve motor learning [48]. Distinct learning phases can be distinguished in motor learning, from an initial "cognitive" phase, which allows to learn motor sequences, to a retention state in which motor performance can be executed in the absence of any practice after long delay [49]. According to some RCTs showing that motor improvements are maintained after few months, AOT is likely to play a key role in achieving the retention state in comparison to motor training only.

Regarding the type of AOT, it might be interesting to understand the differences between the observation of videos and the observation of an operator performing the action. In fact, the only study [29] showing a better improvement in the control than in the AOT group was characterized by the administration of "observation-toimitate" training without videos. Moreover, in this study [29], participants observed the operator and performed actions simultaneously. It is unclear whether such a modality of imitation gives the same effects of that after observation. Indeed, it was shown that even splitting the gesture in the simplest movements during the observation facilitates motor learning [49]. Furthermore, the difference with conventional AOT may be that the observation of actions involves a movement imagination processing before the action execution. Motor imagery, together with AOT, can be considered as a "cognitive rehabilitation tool" and plays a key role in motor learning activating MNS regions that are involved in movement preparation and execution [50]. Finally, the maintenance of attention is probably facilitated during the observation of videos.

An open question is about the role played by the different components of AOT: the observation, the imitation and the combined approach. Only one study [26] (see the paragraph 'Efficacy of AOT') suggested that action observation without imitation produces effects similar to actual action training, probably through the MN activation, but other studies are needed to deeply investigate the neural substrates underlying these mechanisms.

A potential limitation of our study is the risk of a selection bias because papers for this Review were identified through searches of selected databases (see Search Strategy). In addition, only papers published in English were reviewed.

\section{Conclusions}

In conclusion, data presented in the analyzed articles would suggest that AOT is more beneficial than a simple motor training, enhancing motor recovery regardless of the disease. It could be helpful to design more RCTs combining clinical, imaging and neurophysiological evaluations with the aim to correlate clinical motor changes and cerebral plasticity over time in order to deeply understand the mechanisms underlying motor learning after AOT. Further studies with larger samples, longer follow up and correlations with instrumental data are necessary to define the best way to apply AOT in clinical practice.

\section{Additional files}

Additional file 1: Features of included studies. (DOCX $112 \mathrm{~kb}$ )

Additional file 2: Research strategy. (DOCX $13 \mathrm{~kb}$ )

\section{Competing interests}

The authors declare that they have no competing interests.

\section{Author details}

${ }^{1}$ Rehabilitation Department, San Raffaele Scientific Institute, Milan, Italy.

${ }^{2}$ Neuroimaging Research Unit, INSPE, Division of Neuroscience, San Raffaele Scientific Institute, Vita-Salute San Raffaele University, Milan, Italy. ${ }^{3}$ School of Physiotherapy, Vita-Salute San Raffaele University, Milan, Italy. 
Received: 16 May 2015 Accepted: 9 November 2015 Published online: 02 December 2015

\section{References}

1. di Pellegrino G, Fadiga L, Fogassi L, Gallese V, Rizzolatti G. Understanding motor events: a neurophysiological study. Exp Brain Res. 1992;91:176-80.

2. Fadiga L, Fogassi L, Pavesi G, Rizzolatti G. Motor facilitation during action observation: a magnetic stimulation study. J Neurophysiol. 1995;73:2608-11.

3. Lotze M, Montoya P, Erb M, Hülsmann E, Flor H, Klose U, et al. Activation of cortical and cerebellar motor areas during executed and imagined hand movements: an fMRI study. J Cogn Neurosci. 1999;11:491-501.

4. Cattaneo L, Rizzolatti G. The mirror neuron system. Arch Neurol. 2009;66:557-60.

5. Rizzolatti G, Craighero L. The mirror-neuron system. Annu Rev Neurosci. 2004;27:169-92.

6. lacoboni M, Molnar-Szakacs I, Gallese V, Buccino G, Mazziotta JC, Rizzolatti G. Grasping the intentions of others with one's own mirror neuron system. PLoS Biol. 2005;3, e79.

7. Halsband $U$, Lange RK. Motor learning in man: a review of functional and clinical studies. J Physiol Paris. 2006;99:414-24.

8. Mulder T. Motor imagery and action observation: cognitive tools for rehabilitation. J Neural Transm. 2007;114:1265-78.

9. Garrison KA, Winstein CJ, Aziz-Zadeh L. The mirror neuron system: a neural substrate for methods in stroke rehabilitation. Neurorehabil Neural Repair. 2010;24:404-12.

10. Celnik P, Stefan K, Hummel F, Duque J, Classen J, Cohen LG. Encoding a motor memory in the older adult by action observation. Neuroimage. 2006;29:677-84.

11. Stefan K, Cohen LG, Duque J, Mazzocchio R, Celnik P, Sawaki L, et al. Formation of a motor memory by action observation. J Neurosci. 2005;25:9339-46

12. Wang W, Collinger JL, Perez MA, Tyler-Kabara EC, Cohen LG, Birbaumer $N$, et al. Neural interface technology for rehabilitation: exploiting and promoting neuroplasticity. Phys Med Rehabil Clin N Am. 2010;21:157-78.

13. Higgins JPT, Green S. Cochrane Handbook for Systematic Reviews of Interventions Version 5.1.0 [updated March 2011], 2011.

14. Ertelt D, Hemmelmann C, Dettmers C, Ziegler A, Binkofski F. Observation and execution of upper-limb movements as a tool for rehabilitation of motor deficits in paretic stroke patients: protocol of a randomized clinical trial. BMC Neurol. 2012;12:42.

15. Sgandurra G, Ferrari A, Cossu G, Guzzetta A, Biagi L, Tosetti M, et al. Upper limb children action-observation training (UP-CAT): a randomised controlled trial in hemiplegic cerebral palsy. BMC Neurol. 2011;11:80.

16. Altermann CD, Martins AS, Carpes FP, Mello-Carpes PB. Influence of mental practice and movement observation on motor memory, cognitive function and motor performance in the elderly. Braz J Phys Ther. 2014;18:201-9.

17. Cha YJ, Yoo EY, Jung MY, Park SH, Park JH, Lee J. Effects of mental practice with action observation training on occupational performance after stroke. J Stroke Cerebrovasc Dis. 2015;24:1405-13.

18. Ewan LM, Kinmond K, Holmes PS. An observation-based intervention for stroke rehabilitation: experiences of eight individuals affected by stroke. Disabil Rehabil. 2010;32:2097-106.

19. Kim JY, Kim JM, Ko EY. The effect of the action observation physical training on the upper extremity function in children with cerebral palsy. J Exerc Rehabil. 2014;10:176-83.

20. Sugg K, Muller S, Winstein C, Hathorn D, Dempsey A. Does action observation training with immediate physical practice improve hemiparetic upper-limb function in chronic stroke? Neurorehabil Neural Repair. 2015;29:807-17.

21. Taube W, Lorch M, Zeiter S, Keller M. Non-physical practice improves task performance in an unstable, perturbed environment: motor imagery and observational balance training. Front Hum Neurosci. 2010;8:972.

22. Tia B, Mourey F, Ballay Y, Sirandre C, Pozzo T, Paizis C. Improvement of motor performance by observational training in elderly people. Neurosci Lett. 2010;480:138-42.

23. Ertelt D, Small S, Solodkin A, Dettmers C, McNamara A, Binkofski F, et al. Action observation has a positive impact on rehabilitation of motor deficits after stroke. Neuroimage. 2007;36:T164-73.
24. Harmsen WJ, Bussmann JB, Selles RW, Hurkmans HL, Ribbers GM. A mirror therapy-based action observation protocol to improve motor learning after stroke. Neurorehabil Neural Repair. 2015;29:509-16.

25. Kim E, Kim K. Effects of purposeful action observation on kinematic patterns of upper extremity in individuals with hemiplegia. J Phys Ther Sci. 2015;27:1809-11.

26. Lee D, Roh H, Park J, Lee S, Han S. Drinking behavior training for stroke patients using action observation and practice of upper limb function. J Phys Ther Sci. 2013;25:611-4.

27. Franceschini $M$, Ceravolo MG, Agosti M, Cavallini P, Bonassi S, Dall'Armi V, et al. Clinical relevance of action observation in upper-limb stroke rehabilitation: a possible role in recovery of functional dexterity. A randomized clinical trial. Neurorehabil Neural Repair. 2012;26:456-62.

28. Sale P, Ceravolo MG, Franceschini M. Action observation therapy in the subacute phase promotes dexterity recovery in right-hemisphere stroke patients. Biomed Res Int. 2014;457538.

29. Cowles T, Clark A, Mares K, Peryer G, Stuck R, Pomeroy V. Observation-toimitate plus practice could Add little to physical therapy benefits within 31 days of stroke: translational randomized controlled trial. Neurorehabil Neural Repair. 2013;27:173-82.

30. Bang DH, Shin WS, Kim SY, Choi JD. The effects of action observational training on walking ability in chronic stroke patients: a double-blind randomized controlled trial. Clin Rehabil. 2013;27:1118-25.

31. Kim JH, Lee BH. Action observation training for functional activities after stroke: a pilot randomized controlled trial. NeuroRehabilitation. 2013;33:565-74.

32. Kim JS, Kim K. Clinical feasibility of action observation based on mirror neuron system on walking performance in post stroke patients. J Phys Ther Sci. 2012;24:597-9.

33. Park EC, Hwangbo $G$. The effects of action observation gait training on the static balance and walking ability of stroke patients.

J Phys Ther Sci. 2015;27:341-4.

34. Park HR, Kim JM, Lee MK, Oh DW. Clinical feasibility of action observation training for walking function of patients with post-stroke hemiparesis: a randomized controlled trial. Clin Rehabil. 2014;28:794-803.

35. Park CS, Kang KY. The effects of additional action observational training for functional electrical stimulation treatment on weight bearing, stability and gait velocity of hemiplegic patients. J Phys Ther Sci. 2013;25:1173-5.

36. Buccino G, Gatti R, Giusti MC, Negrotti A, Rossi A, Calzetti S, et al. Action observation treatment improves autonomy in daily activities in Parkinson's disease patients: results from a pilot study. Mov Disord. 2011;26:1963-4.

37. Pelosin E, Avanzino L, Bove M, Stramesi P, Nieuwboer A, Abbruzzese G. Action observation improves freezing of gait in patients with Parkinson's disease. Neurorehabil Neural Repair. 2010;24:746-52.

38. Pelosin E, Bove M, Ruggeri P, Avanzino L, Abbruzzese G. Reduction of bradykinesia of finger movements by a single session of action observation in Parkinson disease. Neurorehabil Neural Repair. 2013;27:552-60.

39. Buccino G, Arisi D, Gough P, Aprile D, Ferri C, Serotti L, et al. Improving upper limb motor functions through action observation treatment: a pilot study in children with cerebral palsy. Dev Med Child Neurol. 2012;54:822-8.

40. Sgandurra G, Ferrari A, Cossu G, Guzzetta A, Fogassi L, Cioni G. Randomized trial of observation and execution of upper extremity actions versus action alone in children with unilateral cerebral palsy. Neurorehabil Neural Repair. 2013;27:808-15.

41. Bellelli G, Buccino G, Bernardini B, Padovani A, Trabucchi M. Action observation treatment improves recovery of postsurgical orthopedic patients: evidence for a top-down effect? Arch Phys Med Rehabil. 2010;91:1489-94.

42. Park SD, Song HS, Kim JY. The effect of action observation training on knee joint function and gait ability in total knee replacement patients. J Exerc Rehabil. 2014;10:168-71.

43. Abbruzzese G, Marchese R, Avanzino L, Pelosin E. Rehabilitation for Parkinson's disease: Current outlook and future challenges. Parkinsonism Relat Disord 2015 Epub ahead of print.

44. Perera S, Mody SH, Woodman RC, Studenski SA. Meaningful change and responsiveness in common physical performance measures in older adults. J Am Geriatr Soc. 2006;54:743-9.

45. Beninato M, Gill-Body KM, Salles S, Stark PC, Black-Schaffer RM, Stein J. Determination of the minimal clinically important difference in the FIM instrument in patients with stroke. Arch Phys Med Rehabil. 2006;87:32-9.

46. Buccino G, Vogt S, Ritzl A, Fink GR, Zilles K, Freund HJ, et al. Neural circuits underlying imitation learning of hand actions: an event-related fMRI study. Neuron. 2004;42:323-34. 
47. Press $C$, Heyes C, Kilner JM. Learning to understand others' actions. Biol Lett. $2011 ; 7: 457-60$

48. Kim SS, Kim TH, Lee BH. Effects of action observational training on cerebral hemodynamic changes of stroke survivors: a fTCD study. J Phys Ther Sci. 2014;26:331-4.

49. Gatti R, Tettamanti A, Gough PM, Riboldi E, Marinoni L, Buccino G. Action. observation versus motor imagery in learning a complex motor task: a short review of literature and a kinematics study. Neurosci Lett. 2011;540:37-42.

50. Lotze M, Cohen LG. Volition and imagery in neurorehabilitation. Cogn Behav Neurol. 2006;19:135-40.

Submit your next manuscript to BioMed Central and take full advantage of:

- Convenient online submission

- Thorough peer review

- No space constraints or color figure charges

- Immediate publication on acceptance

- Inclusion in PubMed, CAS, Scopus and Google Scholar

- Research which is freely available for redistribution 